Research Article

\title{
Optimal Selective Maintenance Decision-Making for Consecutive-Mission Systems with Variable Durations and Limited Maintenance Time
}

\author{
Huiying Gao, Xiaoqiang Zhang (iD, Xiaoqiang Yang, and Bo Zheng \\ Aviation Engineering Institute, Civil Aviation Flight University of China, Deyang 618307, China \\ Correspondence should be addressed to Xiaoqiang Zhang; xqzhanguestc@163.com
}

Received 4 February 2021; Revised 16 February 2021; Accepted 19 February 2021; Published 2 March 2021

Academic Editor: Mohammad Yazdi

Copyright (C) 2021 Huiying Gao et al. This is an open access article distributed under the Creative Commons Attribution License, which permits unrestricted use, distribution, and reproduction in any medium, provided the original work is properly cited.

\begin{abstract}
Maintenance is inevitable for repairable components or systems in modern industries. Since the maintenance effectiveness has a great influence on the subsequent operations and in addition, different maintenance options are possible for the components of the system during the break between any two successive missions, the optimal selective maintenance strategy needs to be determined for a system performing successive missions. A number of selective maintenance models were set up on the basis that the durations of each mission are predetermined, the maintenance time is negligible, and the states of the components at the end of the previous mission can be accurately obtained. However, in the actual industrial and military missions, these premises may not always hold. In this paper, a novel selective maintenance model under uncertainties and limited maintenance time is proposed to improve these deficiencies. The genetic algorithm is selected to solve the optimization problem, and an illustrative example is presented to demonstrate the proposed method. The optimal selective maintenance decision without the constraint of maintenance time is used for comparison.
\end{abstract}

\section{Introduction}

Equipment maintenance refers to the technical activities to restore the function of the equipment after deterioration or failures, and it is a key factor affecting the development of industrial, military, aviation, and so on. In general, the maintenance of a component or system can be divided into planned maintenance and unplanned maintenance. The former, also known as preventive maintenance (PM), is usually a maintenance policy formulated in advance. In case of a PM, maintenance activities will be conducted at a preset time or under a predetermined system state. For unplanned maintenance, it is a maintenance policy taken after the system failure occurs and also called corrective maintenance (CM). What the decision-makers usually concern most contains two aspects: one is the reliability of the system after a maintenance activity and the other is the optimal maintenance strategy under limited maintenance time to minimize the maintenance cost. From the early 1960s, academia and industrial community have shown a strong interest in the optimization of maintenance strategies. In the past few decades, a large amount of research works with respect to maintenance have been reported. Scholars have built a number of maintenance models based on the assumption that the components are updated after repairs [1-3]. However, the assumption that the components become brand new after repairs is usually far from the truth. To describe the maintenance situation in the real world, the concept of imperfect maintenance is published. An imperfect maintenance (IM) refers to a maintenance activity after which the system returns to a state between minimal repair and replacement. Alaswad and Xiang [4], Wu and Zuo [5], and Nakagawa [6] have made several comprehensive reviews on imperfect maintenance. In these reviews, some IM models including $(p, q)$ model [7], $(p(t), q(t))$ model [8], Kijima I and Kijima II models $[9,10]$, age reduction models [11], intensity reduction model [12], quasirenewal process model [13], and so on are remarkable. How to 
choose a proper model is an application-dependent and thorny problem and has been recently discussed by Liu et al. [14].

For the moment, the imperfect maintenance has been well studied but how to identify the optimal maintenance strategy under limited maintenance budget and time is still a hot potato for the decision-makers, especially, with the addition of uncertainties. The first reported work with respect to selective maintenance should date back to Rice et al. [15]. Immediately after that, Cassady et al. [16, 17] proposed some selective maintenance problems and a general selective maintenance model was constructed by considering different maintenance actions. In management science, selective maintenance is a class of optimization problems in nature. Rice et al. [15] and Rajagopalan and Cassady [18] proposed several enumerating methods to resolve the selective maintenance models, but they become computationally inefficient as the crescent complexity of selective maintenance models. Therefore, some heuristic algorithms such as simulated annealing algorithm [19], genetic algorithm [20,21], ant colony optimization [22], and artificial neural networks [23] have been applied to identify an optimal maintenance strategy under acceptable computational efficiency.

In practice, components or systems may be connected to each other in an arbitrary form and the components may be multistate. Therefore, Liu and Huang [24], Pandey et al. [25], and Liu et al. [26] studied the optimal selective maintenance strategy for a multistate system consisting of binary-state components or multistate components, respectively. Dao and Zuo [27] proposed a selective maintenance model of multistate systems based on the load-dependent degradation model. Besides, some selective maintenance optimizations based on various IM models such as the Kijima I model [28], the Kijima II model [24], and the hybrid model [29] have also been studied.

Although a lot of research with respect to selective maintenance models have be done, but most of the models were set up on the basis that the durations of each mission were predetermined and the states of the components at the end of the previous mission can be accurately obtained. In the actual industrial tasks and military missions, these premises may not always hold. For example, an airplane may change the flight course according to the weather or the commands from air traffic controllers. In these situations, the mission duration will be variable. Jiang and Liu [30] and Khatab et al. [31] set up some selective maintenance models considering the uncertainties associated with the durations of missions but they do not concern the constraint of maintenance time. In this paper, a novel selective maintenance model is proposed under variable mission durations and limited maintenance time.

The remainder of this paper is organized as follows. Section 2 lists some hypotheses on which the selective maintenance model is set up, and imperfect maintenance based on the Kijima II model is introduced. Section 3 discusses the selective maintenance model under uncertainties. Section 4 presents an illustrative example. Conclusions are finally summarized in Section 5.

\section{Model Hypotheses and Imperfect Maintenance Based on Kijima II Model}

In military operations, a weapon may change mission mode according to the actual situation and the mission durations are correspondingly variable. To maintain the warfare capability, the maintenance activities must maximize the reliability of the system within limited maintenance time. In this paper, to set up the novel selective maintenance model, some basic hypotheses are made as follows:

(1) The system for selective maintenance is composed of $n$ repairable components in any combination ways. The states of all the components as well as the system can be identified at any time and under a binary-state (functioning or failed).

(2) As shown in Figure 1, the maintenance activities can be only conducted in the break between any two successive missions and the duration of each break is a limited constant, denoting as $T^{M}$.

(3) Several maintenance options are available for components during the breaks, including do nothing $(\mathrm{DN})$, minimal repair (MR), replacement maintenance $(\mathrm{RM})$, and imperfect preventive/corrective maintenance (IPM/ICM).

(4) The state of element $i$ at the beginning and the end of the $k^{\text {th }}$ mission can be described by binary variables $S_{i, k}^{B}$ and $S_{i, k}^{E}$, respectively. Moreover, it has

$$
\begin{aligned}
& S_{i, k}^{B}= \begin{cases}1, & \text { if component is functioning, } \\
0, & \text { otherwise }\end{cases} \\
& S_{i, k}^{E}= \begin{cases}1, & \text { if component is functioning, } \\
0, & \text { otherwise }\end{cases}
\end{aligned}
$$

(5) All the components are brand new before the first mission and the duration of the first mission is a given constant $t_{1}$, the state of element $i$ ( $i \in\{1,2, \cdots$, $n\})$ at the end of the first mission is known as $S_{i, 1}^{E}$, and the virtual age of each element at the end of the first mission is also predetermined, whereas it is uncertain for the subsequent missions.

(6) The duration of each future mission (except the first mission) can be denoted as a discrete variable $T_{k}$ $(k \in\{2, \cdots, N\})$ in this paper, which has $w_{k}$ possible values and the probability for the $j^{\text {th }}(j \in\{1,2, \cdots$, $\left.\left.w_{k}\right\}\right)$ possible value $t_{k}^{j}$ is $p_{k}^{j}$.

For component $i(i \in\{1,2, \cdots n\})$, it is assumed that the virtual ages at the beginning and the end of the $k^{\text {th }}$ mission are $V_{i, k}^{B}$ and $V_{i, k}^{E}$, respectively; the virtual age after the $k^{\text {th }}(k \in\{1,2, \cdots, N-1\})$ maintenance is $V_{i, k}^{M}$ (obviously, $V_{i, k+1}^{B}=V_{i, k}^{M}$ and the minimum is zero); the failure time of component $i$ from the beginning of the $k^{\text {th }}$ mission is represented by $T_{i, k}^{F}\left(T_{i, k}^{F} \in[0,+\infty)\right)$; the actual service time of component $i$ in the $k^{\text {th }}$ mission can be denoted by a random variable $T_{i, k}^{\Theta}\left(T_{i, k}^{\Theta} \in\left[0, t_{k}\right]\right)$, where $t_{k}$ is the duration of the $k^{\text {th }}$ mission. Then, it is obvious that $T_{i, k}^{\Theta} \in\left(0, t_{k}\right)$ in the case that the component fails in the $k^{\text {th }}$ mission; otherwise, 


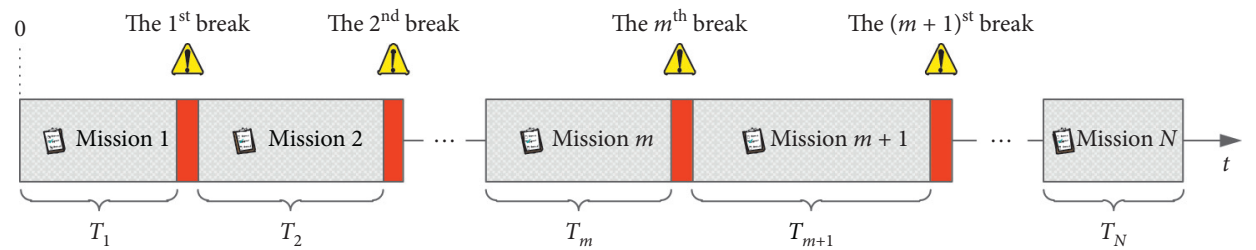

Figure 1: The successive missions and breaks.

the component works properly all through the $k^{\text {th }}$ mission and it has $T_{i, k}^{\Theta}=t_{k}$. In addition, since the maintenance activity can only be conducted during the break between the two successive missions, it is easy to gain the following:

$$
V_{i, k}^{E}=V_{i, k}^{B}+T_{i, k}^{\Theta} \text {. }
$$

According to the Kijima II model and equation (2), the virtual age of component $i$ after the $k^{\text {th }}$ maintenance can be defined as follows:

$$
V_{i, k+1}^{B}=\beta_{i, k} V_{i, k}^{E}=\beta_{i, k}\left(V_{i, k}^{B}+T_{i, k}^{\Theta}\right),
$$

where $\beta_{i, k}\left(\beta_{i, k} \in[0,1]\right)$ is the age reduction factor, and it is closely related to the maintenance effectiveness.

In maintenance activities, the maintenance cost can be roughly divided into the fixed maintenance cost and the reparative maintenance cost. As a result, the total maintenance cost of component $i$ allocated in the $k^{\text {th }}$ break can be expressed as

$$
C_{i, k}=C_{i, k}^{0}+C_{i, k}^{M}
$$

where $C_{i, k}^{0}$ represents the inevitable fixed cost such as the payment for the serviceman and $C_{i, k}^{M}$ represents the reparative maintenance cost used to reduce the virtual age of component $i$ and it is controllable.

Generally, the more reparative maintenance cost is, the better or more effective the maintenance will be. A great number of studies with respect to the age reduction factor can be found. Diallo et al. [32] indicated that the reparative maintenance cost and the current virtual age of the component were the two principal factors affecting the degree of system repair. For convenience, the maintenance cost for performing an minimal repair (MR), an maximum imperfect maintenance (MIM), and a replacement maintenance (RM) are denoted by $C_{i, k}^{\mathrm{MR}}, C_{i, k}^{\mathrm{MIM}}$, and $C_{i, k}^{\mathrm{RM}}$ hereinafter, respectively. An ICM procedure for a failed component can be split up into an MR and a further maintenance to reduce the virtual age. Therefore, performing an MIM to a failed component is more expensive compared with a functioning component. When $C_{i, k}^{M}=C_{i, k}^{\mathrm{MR}}, \mathrm{MR}$ is performed, the degree of repair of the component is zero, and the repaired component is restored to "as bad as old (ABAO)"; if $C_{i, k}=0$, then it corresponds to DN. Particularly, if $C_{i, k}^{M}=C_{i, k}^{\mathrm{RM}}$, the repaired component is restored to "as good as new (AGAN)." It is easy to understand that when the virtual age of the component is small, the reparative cost of maintenance is relatively low and it can achieve a good maintenance effect, whereas, as the virtual age of the component continues to increase, the cost of reparative maintenance will increase and the corresponding maintenance effect will gradually decrease meanwhile. Nguyen et al. [33] described the relationships among preventive maintenance cost, replacement maintenance cost, and the degree of rejuvenation after system repair. Pandey et al. [29] defined the age reduction factor as a function related to maintenance cost as follows:

$$
\beta_{i, k}=\left\{\begin{array}{l}
1-\left(\frac{C_{i, k}^{M}-C_{i, k}^{\mathrm{MR}}}{C_{i, k}^{\mathrm{MIM}}}\right)^{\left(1 / m_{i, k}\right)}, \quad \text { for } S_{i, k}^{E}=0 \text { and } C_{i, k}^{M} \in\left[C_{i, k}^{\mathrm{MR}}, C_{i, k}^{\mathrm{MR}}+C_{i, k}^{\mathrm{MIM}}\right], \\
1-\left(\frac{C_{i, k}^{M}}{C_{i, k}^{\mathrm{MIM}}}\right)^{\left(1 / m_{i, k}\right)}, \quad \text { for } S_{i, k}^{E}=10 \text { and } C_{i, k}^{M} \in\left[0, C_{i, k}^{\mathrm{MIM}}\right],
\end{array}\right.
$$

where $m_{i, k}$ is the characteristic constant and it determines the exact relationship between the reparative maintenance cost $C_{i, k}^{M}$ and the age reduction factor $\beta_{i, k}$. As shown in Figure 2 , if the component is in functioning state, when the ratio between $C_{i, k}^{M}$ and $C_{i, k}^{\mathrm{MIM}}$ is a constant, the variable $m_{i, k}$ will lead to different age reduction factors.

From Figure 2, it can be found that the larger the value of $m_{i, k}$ is, the more obvious the maintenance effect is. As discussed in $[26,34]$, this scenario corresponds to the component with smaller virtual age; whereas, the value of $m_{i, k}$ decreases as the component aging and the effect would be decreased for even a large amount of maintenance cost. Pandey et al. [29] defined the characteristic constant $m_{i, k}$, the mean residual life (MRL) $T_{i, k}^{\mathrm{MRL}}$, and the virtual age $V_{i, k}^{E}$ and discussed the relationship of the three, and it has the following:

$$
m_{i, k}=\frac{T_{i, k}^{\mathrm{MRL}}}{V_{i, k}^{E}} .
$$




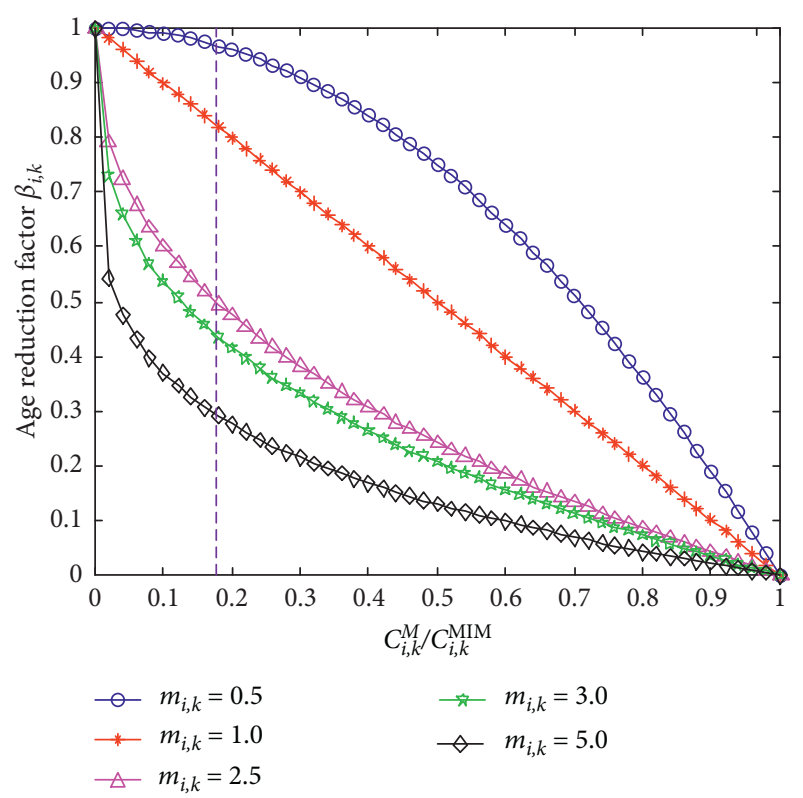

FIgURE 2: The relationship between $\beta_{i, k}$ and $\left(C_{i, k}^{M} / C_{i, k}^{\mathrm{MIM}}\right)$.

Assuming that the virtual age $V_{i, k}^{B}$ at the beginning of the $k^{\text {th }}$ mission is a given constant $v_{i, k}^{b}$, the actual service time in the $k^{\text {th }}$ mission is $t^{\Theta}$, then the mean residual life $T_{i, k}^{\mathrm{MRL}}$ can be calculated as follows:

$$
T_{i, k}^{\mathrm{MRL}}=\frac{\int_{t^{\Theta}}^{+\infty} R_{i, k}\left(x \mid v_{i, k}^{b}\right) \mathrm{d} x}{R_{i, k}\left(t^{\Theta} \mid v_{i, k}^{b}\right)},
$$

where $R_{i, k}\left(t^{\Theta} \mid v_{i, k}^{b}\right)$ is a conditional probability, and it represents the survival probability of component $i$ at the end of the $k^{\text {th }}$ mission under the condition that $V_{i, k}^{B}=v_{i, k}^{b}$.

Substituting equation (7) into equation (6),

$$
m_{i, k}=\frac{T_{i, k}^{\mathrm{MRL}}}{V_{i, k}^{E}}=\frac{\int_{t^{\Theta}}^{+\infty} R_{i, k}\left(x \mid v_{i, k}^{b}\right) \mathrm{d} x}{\left(v_{i, k}^{b}+t^{\Theta}\right) R_{i, k}\left(t^{\Theta} \mid v_{i, k}^{b}\right)} .
$$

In equation (8), if $T_{i, k}^{\mathrm{MRL}} \geq V_{i, k}^{E}$, i.e., $m_{i, k} \geq 1$, the virtual age at the end of the $k^{\text {th }}$ mission is less than half of the mean residual life under the condition that $V_{i, k}^{B}=v_{i, k}^{b}$, then component $i$ is young; whereas, if $T_{i, k}^{\mathrm{MRL}}<V_{i, k}^{E}$, i.e., $m_{i, k}<1$, then component $i$ is in aging state and the performance of the component will not be significantly improved even more maintenance cost is allocated.

\section{The Proposed Selective Maintenance Model under Uncertainties and Limited Maintenance Time}

The survival probability and the virtual age of component $i$ at the end of the $k^{\text {th }}$ mission are closely related to its state at the beginning of the $k^{\text {th }}$ mission. The detailed evaluation method will be discussed in the following:

(a) $S_{i, k}^{B}=0$.
In this case, component $i$ is failed at the beginning of the $k^{\text {th }}$ mission. Since the maintenance activities can only be conducted in the breaks between any two successive missions, as a result, component $i$ does not work in reality during this mission, and the survival probability at the end of the $k^{\text {th }}$ mission as well as the incremental virtual age of component $i$ throughout this mission are both zero.

(b) $S_{i, k}^{B}=1$.

In this case, component $i$ is in functioning state at the beginning of the $k^{\text {th }}$ mission. According to the state of component $i$ at the end of the $k^{\text {th }}$ mission, two cases can be subdivided further.

(b1) $S_{i, k}^{B}=1, S_{i, k}^{E}=1$.

In this case, component $i$ is operating smoothly during the $k^{\text {th }}$ mission and it remains in functioning state at the end of the $k^{\text {th }}$ mission. Therefore, the survival probability of component $i$ in the $k^{\text {th }}$ mission, under the condition that $V_{i, k}^{B}=v_{i, k}^{b}$, can be denoted as a conditional probability $R_{i, k}\left(t \mid v_{i, k}^{b}\right)$, which satisfies the following:

$$
R_{i, k}\left(t \mid v_{i, k}^{b}\right)=\frac{R_{i}\left(t+v_{i, k}^{b}\right)}{R_{i}\left(v_{i, k}^{b}\right)}
$$

where $t$ is the time interval since the beginning of the $k^{\text {th }}$ mission and $R_{i}\left(v_{i, k}^{b}\right)$ is the survival probability of component $i$ in the case that the virtual age is $v_{i, k}^{b}$. Since component $i$ is in functioning state at the end of the $k^{\text {th }}$ mission, the actual service time for component $i$ throughout the $k^{\text {th }}$ mission satisfies $T_{i, k}^{\Theta}=T_{k}=$ $t_{k}$. The virtual age and the survival probability at the end of the $k^{\text {th }}$ mission can be expressed as $V_{i, k}^{E}=v_{i, k}^{b}+t_{k}$ and $R_{i, k}\left(t_{k} \mid v_{i, k}^{b}\right)$, respectively.

(b2) $S_{i, k}^{B}=1, S_{i, k}^{E}=0$.

On the contrary, component $i$ may fail within the $k^{\text {th }}$ mission. In this case, the value of failure time $T_{i, k}^{\Theta}$ lies in the time interval $\left(0, t_{k}\right)$, i.e., $T_{i, k}^{\Theta} \in\left(0, t_{k}\right)$. The probability density function (PDF) of $T_{i, k}^{\Theta}$ under the condition that $V_{i, k}^{B}=$ $v_{i, k}^{b}, T_{k}=t_{k}$ can be calculated by the following:

$f_{T_{i, k}^{\Theta} \mid V_{i, k}^{B}, T_{k}}\left(t \mid v_{i, k}^{b}, t_{k}\right)=\frac{f_{T_{i, k}^{\Theta} \mid V_{i, k}^{B}}\left(t \mid v_{i, k}^{b}\right)}{1-R_{i, k}\left(t_{k} \mid v_{i, k}^{b}\right)}, \quad 0<t<t_{k}$,

where $f_{T_{i, k}^{\Theta} \mid V_{i, k}^{B}}\left(t \mid v_{i, k}^{b}\right)$ is the PDF of failure time $T_{i, k}^{\Theta}$. Then, the virtual age of component $i$ at the end of the $k^{\text {th }}$ mission is $V_{i, k}^{E}=V_{i, k}^{B}+T_{i, k}^{\Theta}$; furthermore, the cumulative distribution function (CDF) and PDF of $V_{i, k}^{E}$ can be derived as follows: 


$$
\begin{aligned}
& F_{V_{i, k}^{E} \mid V_{i, k}^{B}, T_{k}}\left(v_{i, k}^{e} \mid v_{i, k}^{b}, t_{k}\right)=F_{T_{i, k}^{\Theta} \mid V_{i, k}^{B}, T_{k}}\left(v_{i, k}^{e}-v_{i, k}^{b} \mid v_{i, k}^{b}, t_{k}\right), \\
& f_{V_{i, k}^{E} \mid V V_{i, k}^{B}, T_{k}}\left(v_{i, k}^{e} \mid v_{i, k}^{b}, t_{k}\right)=\frac{\mathrm{d} F_{T_{i, k}^{\Theta} \mid V_{i, k}^{B}, T_{k}}\left(v_{i, k}^{e}-v_{i, k}^{b} \mid v_{i, k}^{b}, t_{k}\right)}{\mathrm{d} v_{i, k}^{e}}=f_{T_{i, k}^{\Theta} \mid V_{i, k}^{B}, T_{k}}\left(v_{i, k}^{e}-v_{i, k}^{b} \mid v_{i, k}^{b}, t_{k}\right),
\end{aligned}
$$

where $F_{T_{i, k}^{\Theta} \mid V_{i, k}^{B}, T_{k}}\left(v_{i, k}^{e}-v_{i, k}^{b} \mid v_{i, k}^{b}, t_{k}\right)$ and $f_{T_{i, k}^{\Theta} \mid V_{i, k}^{B}, T_{k}}$ $\left(v_{i, k}^{e}-v_{i, k}^{b} \mid v_{i, k}^{b,}, t_{k}\right)$ are the CDF and PDF of $T_{i, k}^{\Theta}$, respectively; $\quad F_{V_{i, k}^{E} \mid V_{i, k}^{B}, T_{k}}\left(v_{i, k}^{e} \mid v_{i, k}^{b}, t_{k}\right) \quad$ and $f_{V_{i, k}^{E} \mid V_{i, k}^{B}, T_{k}}\left(v_{i, k}^{e} \mid v_{i, k}^{b}, t_{k}\right)$ are the CDF and PDF of $V_{i, k}^{E}$, respectively.
If ICM is selected for a failed component $i$, it will be restored to functioning state after the maintenance activity and it has $S_{i, k+1}^{B}=1, \quad V_{i, k+1}^{B}=\beta_{i, k}\left(V_{i, k}^{B}+T_{i, k}^{\Theta}\right), \quad\left(\beta_{i, k} \in\right.$ $[0,1))$; in this scenario, the CDF and PDF of $V_{i, k+1}^{B}$ under the condition that $V_{i, k}^{B}=v_{i, k}^{b}, T_{k}=t_{k}$ can be expressed as follows:

$$
\begin{aligned}
& F_{V_{i, k+1}^{B} \mid V_{i, k}^{B}, T_{k}}\left(v_{i, k+1}^{b} \mid v_{i, k}^{b}, t_{k}\right)=F_{T_{i, k}^{\Theta} \mid V_{i, k}^{B}, T_{k}}\left(v_{i, k+1}^{b} / \beta_{i, k}-v_{i, k}^{b} \mid v_{i, k}^{b}, t_{k}\right), \\
& f_{V_{i, k+1}^{B} \mid V_{i, k}^{B}, T_{k}}\left(v_{i, k+1}^{b} \mid v_{i, k}^{b}, t_{k}\right)=\frac{\mathrm{d} F_{T_{i, k}^{\Theta} \mid V V_{i, k}^{B}, T_{k}}\left(v_{i, k+1}^{b} / \beta_{i, k}-v_{i, k}^{b} \mid v_{i, k}^{b}, t_{k}\right)}{\mathrm{d} v_{i, k+1}^{b}}=\frac{f_{T_{i, k}^{\Theta} \mid V_{i, k}^{B}, T_{k}}\left(v_{i, k+1}^{b} / \beta_{i, k}-v_{i, k}^{b} \mid v_{i, k}^{b}, t_{k}\right)}{\beta_{i, k}},
\end{aligned}
$$

where $F_{V_{i, k+1}^{B} \mid V_{i, k}^{B}, T_{k}}\left(v_{i, k+1}^{b} \mid v_{i, k}^{b}, t_{k}\right)$ and $f_{V_{i, k+1}^{B} \mid V_{i, k}^{B}, T_{k}}\left(v_{i, k+1}^{b} \mid v_{i, k}^{b}, t_{k}\right)$ are the CDF and PDF of $V_{i, k+1}^{B}$, respectively.

In a number of actual industrial and military environments, the durations are variables, together with the inherent randomness of the failure time $T_{i, k}^{F}$; as a result, the actual service time $T_{i, k}^{\Theta}$ is a random variable. In addition to these uncertainties, the virtual age at the beginning of the $k^{\text {th }}$ mission and the age reduction factor $\beta_{i, k}$ associated with the maintenance options are uncertain; thus, the virtual age at the beginning of the $(k+1)^{\mathrm{st}}$ mission is stochastic. The propagation of a variety of uncertainties between the successive missions is illustrated in Figure 3.

The survival probability of component $i$ in the $k^{\text {th }}$ mission under the condition that $\mathbf{T}_{k-1}=\mathbf{t}_{k-1}=\left(t_{1}, t_{2}, \cdots\right.$, $\left.t_{k-1}\right)$ can be expressed as follows:

$$
R_{i, k}\left(t \mid \mathbf{t}_{k-1}\right)=\operatorname{Pr}\left\{T_{i, k}>t \mid T_{1}=t_{1}, T_{2}=t_{2}, \cdots, T_{k-1}=t_{k-1}\right\} .
$$

Similarly, the reliability for component $i$ in successfully completing the $k^{\text {th }}$ mission under the condition that $\mathbf{T}_{k}=$ $\mathbf{t}_{k}=\left(t_{1}, t_{2}, \cdots, t_{k}\right)$ can be denoted as follows:

$$
\begin{aligned}
R_{i, k}\left(\mathbf{t}_{k}\right) & =\operatorname{Pr}\left\{S_{i, k}^{E}=1 \mid T_{1}=t_{1}, T_{2}=t_{2}, \cdots, T_{k}=t_{k}\right\} \\
& =R_{i, k}\left(t_{k} \mid \mathbf{t}_{k-1}\right),
\end{aligned}
$$

where $t$ is the elapsed time since the beginning of the $k^{\text {th }}$ mission.

In case of $\mathbf{T}_{k}=\mathbf{t}_{k}=\left(t_{1}, t_{2}, \cdots, t_{k}\right)$, the reliability of the system in successfully completing the $k^{\text {th }}$ mission satisfies the following:

$$
\begin{aligned}
R_{s, k}\left(\mathbf{t}_{k}\right) & =R_{s, k}\left(t_{1}, t_{2}, \cdots, t_{k}\right) \\
& =\operatorname{Pr}\left\{S_{s, k}^{E}=1 \mid T_{1}=t_{1}, T_{2}=t_{2}, \cdots, T_{k}=t_{k}\right\},
\end{aligned}
$$

where $S_{s, k}^{E}=1$ indicates that the system is in functioning state at the end of the $k^{\text {th }}$ mission.
According to the sixth hypothesis in Section 2, the durations in this study are assumed to be discrete variables. Consequently, the reliability of the system in successfully completing the $k^{\text {th }}$ mission can be derived on the basis of equation (17) as follows:

$$
\begin{aligned}
R_{s, k}^{D} & =\operatorname{Pr}\left\{S_{s, k}^{E}=1\right\} \\
& =\sum_{j_{1}=1}^{w_{1}} p_{1}^{j_{1}} \sum_{j_{2}=1}^{w_{2}} p_{2}^{j_{2}} \cdots \sum_{j_{k}=1}^{w_{k}} p_{k}^{j_{k}} R_{s, k}\left(t_{1}^{j_{1}}, t_{2}^{j_{2}}, \cdots, t_{k}^{j_{k}}\right) \\
& =\sum_{j_{1}=1}^{w_{1}} \sum_{j_{2}=1}^{w_{2}} \cdots \sum_{j_{k}=1}^{w_{k}}\left[\left(\prod_{v=1}^{k} p_{v}^{j_{v}}\right) R_{s, k}\left(t_{1}^{j_{1}}, t_{2}^{j_{2}}, \cdots, t_{k}^{j_{k}}\right)\right],
\end{aligned}
$$

where $w_{v}(v \in\{1,2, \cdots, k\})$ and $t_{v}^{j_{v}}\left(j_{v} \in\left\{1,2, \cdots, w_{v}\right\}\right)$ are the number of possible values and the $j_{v}$ th possible value for $t_{v}$, respectively, and $p_{v}^{j_{v}}$ is the corresponding probability mass.

The purpose of the selective maintenance is to maximize the probability for completing all the missions subject to the total maintenance budget. According to the Hypothesis 2, the first maintenance activity is conducted after mission 1 is over. In other words, the maintenance activities have nothing to do with $R_{s, 1}^{D}$. Thus, the selective optimization model can be simplified as follows:

$$
\begin{array}{ll}
\max & R_{s}=\prod_{k=2}^{N} R_{s, k}^{D}, \\
& \sum_{j=1}^{N-1} \sum_{i=1}^{n} C_{i, j} \leq C^{\delta} ; \\
\text { s.t. } & 0 \leq C_{i, j} \leq C_{i, j}^{0}+C_{i, j}^{\mathrm{RM}} ; \\
& \sum_{i=1}^{n} t_{i, j}^{M} \leq T^{M} ; \\
& i \in\{1,2, \cdots, n\} ; j \in\{1,2, \cdots, N-1\} .
\end{array}
$$




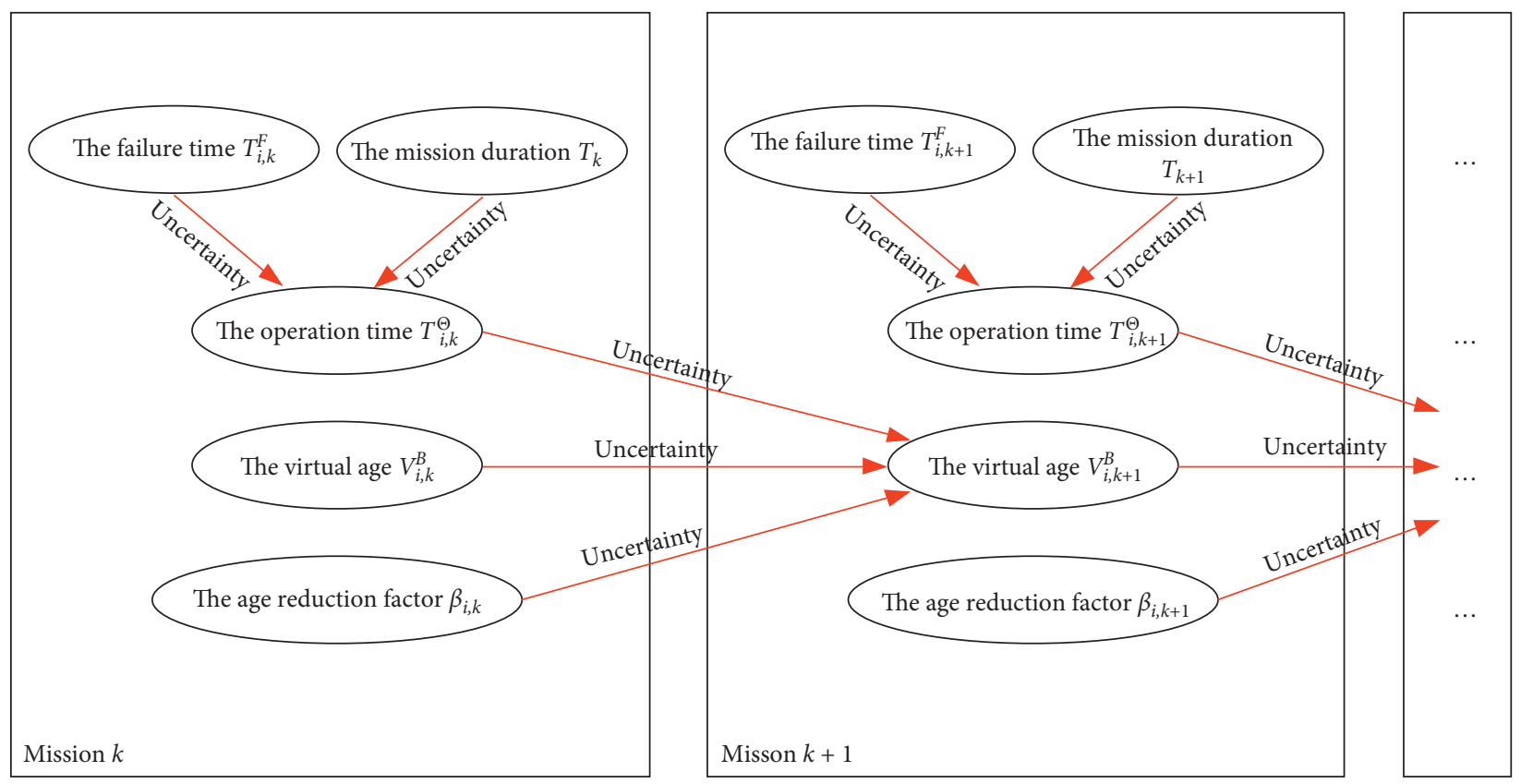

FIGURE 3: The propagation of uncertainty between the successive missions.

where $C_{i, k}$ is the maintenance cost allocated to component $i$ in the $k^{\text {th }}(k \in\{1,2, \cdots, N-1\})$ break, and $C^{\delta}$ is the limited maintenance budget for all the maintenance activities.

According to equation (19), the selective maintenance decision is a complex nonlinear programming problem with continuous decision variables. It is scarcely possible to solve the problem within the limited time by traditional reliability analysis methods or optimization algorithms [35-40]. However, some advanced intelligent optimization algorithms such as genetic algorithm, simulated annealing algorithm, Bayesian networks [41-43], and ant colony algorithm can find the global approximate optimal solution in a reasonable amount of time. Without loss of generality, the widely used genetic algorithm is selected in this study.

The key point to solve optimization problems with the genetic algorithm lies in how to convert the feasible solutions into population individuals in the genetic algorithm. Theoretically, the maintenance cost allocated to each component can be any real number in the feasible region. To reduce the computational complexity, the decision variables are converted into integer variables and the feasible solutions corresponding to the population individuals are expressed as an integer vector $\mathbf{L}$ composed of $n \times(N-1)$ elements and yields the following:

$$
\mathbf{L}=(\underbrace{l_{1,1}, l_{2,1}, \cdots, l_{n, 1}}_{\text {the } 1^{\text {st }} \text { break }}, \cdots, \underbrace{l_{1, k}, l_{2, k}, \cdots, l_{n, k}}_{\text {the } k^{\text {th }} \text { break }}, \cdots, \underbrace{l_{1, N-1}, l_{2, N-1}, \cdots, l_{n, N-1}}_{\text {the }(N-1)^{\text {th }} \text { break }}),
$$

where $l_{i, k}\left(0 \leq l_{i, k} \leq W_{L}\right)$ is the maintenance level for component $i$ in the $k^{\text {th }}$ break and $W_{L}$ is the number of maintenance levels determined by decision-makers. It is easy to understand that the larger $W_{L}$ is, the more accurate the optimal solutions will be while more computation complexity. Therefore, the decision-makers need to make a compromise between the accuracy and the computation complexity. The different values of $W_{L}$ correspond to various maintenance costs and maintenance times. The detailed information with respect to maintenance activities is tabulated in Table 1.

The process of the optimization for selective maintenance based on the genetic algorithm is illustrated with Figure 4 . It contains mainly six steps, and the pseudoprogram can described as follows.
Step 1. Initialization: set $k=1$; determine the system structure as well as the initial state and the virtual age of each component at the end of the first mission;

Step 2. Feasible population: generate initial feasible individuals according to the number of population individuals set by the genetic algorithm;

Step 3. Virtual age and survival probability for component: convert the population individuals into the maintenance cost allocated to each component; calculate the virtual age $V_{i, k}^{E}$ and the survival probability $R_{i, k}\left(\mathbf{t}_{k}\right)$ for each component;

Step 4. Survival probability and maintenance cost for the system: evaluate the survival probability $R_{s, k}^{\Delta}$ 
TABLE 1: The maintenance actions and relevant information corresponding to different maintenance levels.

\begin{tabular}{|c|c|c|c|c|}
\hline \multirow[b]{2}{*}{ Levels $\left(l_{i, k}\right)$} & \multirow[b]{2}{*}{ Costs $\left(C_{i, k}\right)$} & \multirow{2}{*}{ Time $\left(t_{i, k}^{M}\right)$} & \multicolumn{2}{|c|}{ Maintenance activities } \\
\hline & & & $V_{i, k}^{E}=0$ & $V_{i, k}^{E}=1$ \\
\hline 0 & 0 & 0 & $\mathrm{DN}$ & $\mathrm{DN}$ \\
\hline 1 & $C_{i, k}^{0}+C_{i, k}^{\mathrm{MR}}$ & $t_{i, k}^{M}(1)$ & MR & IPM \\
\hline$\vdots$ & 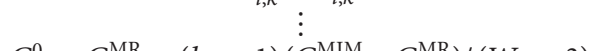 & & $\vdots$ & $\vdots$ \\
\hline $\begin{array}{l}l_{i, k} \\
\vdots\end{array}$ & $\begin{array}{c}C_{i, k}^{0}+C_{i, k}^{\mathrm{MR}}+\left(l_{i, k}-1\right)\left(C_{i, k}^{\mathrm{MIM}}-C_{i, k}^{\mathrm{MR}}\right) /\left(W_{L}-3\right) \\
\vdots\end{array}$ & $\begin{array}{c}t_{i, k}^{M}\left(l_{i, k}\right) \\
\vdots\end{array}$ & $\begin{array}{l}\text { ICM } \\
\vdots\end{array}$ & $\begin{array}{l}\text { IPM } \\
\quad \vdots\end{array}$ \\
\hline$W_{L}-2$ & $C_{i, k}^{0}+C_{i, k}^{\mathrm{MIM}}$ & $t_{i, k}^{M}\left(W_{L}-2\right)$ & ICM & MIM \\
\hline$W_{L}-1$ & $C_{i, k}^{0}+C_{i, k}^{\mathrm{MR}}+C_{i, k}^{\mathrm{MIM}}$ & $t_{i, k}^{M}\left(W_{L}-1\right)$ & MIM & MIM \\
\hline$W_{L}$ & $C_{i, k}^{0}+C_{i, k}^{\mathrm{RM}}$ & $t_{i, k}^{M}\left(W_{L}\right)$ & $\mathrm{RM}$ & $\mathrm{RM}$ \\
\hline
\end{tabular}

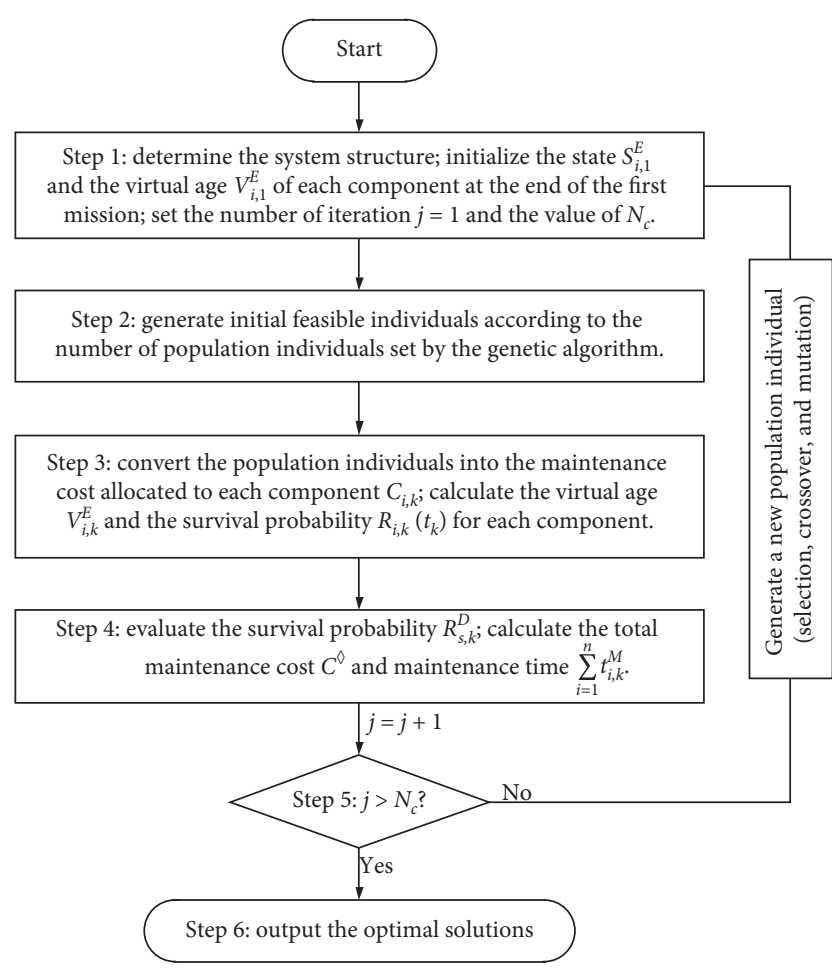

FIGURE 4: The process of the genetic algorithm.

according to the system structure; calculate the actual total maintenance cost $C^{\delta}$;

Step 5. Stopping criterion: if the number of iteration optimizations is less than the specified value $N_{c}$, a new population individual will be generated according to the selection, crossover, and mutation operation rules of the genetic algorithm, and the process goes to step 3; otherwise, the process proceeds to step 6;

Step 6. End: output the optimal solutions.

\section{An Illustrative Example}

To demonstrate the feasibility of the proposed method, an illustrative example with respect to selective maintenance will be studied in this section. The hydraulic system of an airplane is composed of three components, as shown in Figure 5, and it is going to perform four different flights (four successive missions). Assuming that all the components are in brand new state at the beginning of the first mission, the lifetime of

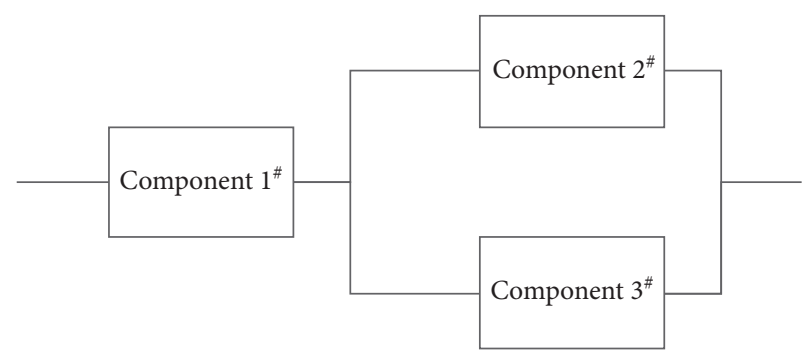

FIgURE 5: The reliability logic block diagram for the hydraulic system.

TABLE 2: The parameters of each component (unit of maintenance cost: $¥ 1,000$; unit of time: day).

\begin{tabular}{ccccccccccc}
\hline$i$ & $\eta_{i}$ & $\gamma_{i}$ & $S_{i, 1}^{B}$ & $S_{i, 1}^{E}$ & $V_{i, 1}^{B}$ & $T_{i, 1}^{\Theta}$ & $C_{i, k}^{0}$ & $C_{i, k}^{\mathrm{MR}}$ & $C_{i, k}^{\mathrm{MIM}}$ & $C_{i, k}^{\mathrm{RM}}$ \\
\hline 1 & 22 & 2.5 & 1 & 1 & 0 & 6.5 & 0.28 & 1.6 & 28 & 37 \\
2 & 16 & 1.8 & 1 & 1 & 0 & 6.5 & 0.31 & 2.6 & 32 & 40 \\
3 & 18 & 3.2 & 1 & 0 & 0 & 3.5 & 0.42 & 2.2 & 24 & 35 \\
\hline
\end{tabular}

each component follows a Weibull distribution, the scale parameter $\eta_{i}(i \in\{1,2,3\})$, and the shape parameter $\gamma_{i}(i \in\{1,2,3\})$ as well as the maintenance cost, and the state and the virtual age at the end of the first mission are tabulated in Table 2. The maintenance times corresponding to different maintenance levels are tabulated in Table 3. The durations of the four missions are discrete variables, and the probability distributions are tabulated in Table 4. In this study, the maximum maintenance level $W_{L}$ is set as $W_{L}=5$, the total budget for maintenance cost $C^{\delta}$ is $240,000 \mathrm{CNY}$, and the maximum allowable maintenance time is 6 hour. The optimal maintenance decision can be obtained, and it is tabulated in Table 5. Scenario 1 denotes that the limited maintenance time is considered in the optimal selective maintenance decision, whereas Scenario 2 does not. The optimal selective maintenance decisions in Scenario 2 are used for comparison.

It can be seen from Table 5 that there are three selective maintenance decisions need to be made for the four missions. Empirically, the more maintenance cost is allocated to a component, the more reliable it is. The maximal reliability of the system in successfully completing the missions under two different scenarios is 0.8497 and 0.8883 , respectively. It is easy to see that the maintenance cost in Scenario 1 is larger than the cost in Scenario 2, but the reliability is worse. The main reason for this result is the limited maintenance time. 
TABLE 3: The maintenance time corresponding to different maintenance levels (unit of time: hour).

\begin{tabular}{|c|c|c|}
\hline Component ID & Maintenance level & Time \\
\hline \multirow{6}{*}{$1^{\#}$} & 0 & 0 \\
\hline & 1 & 0.50 \\
\hline & 2 & 3.20 \\
\hline & 3 & 4.40 \\
\hline & 4 & 5.10 \\
\hline & 5 & 0.60 \\
\hline \multirow{6}{*}{$2^{\#}$} & 0 & 0 \\
\hline & 1 & 0.20 \\
\hline & 2 & 3.20 \\
\hline & 3 & 4.80 \\
\hline & 4 & 5.31 \\
\hline & 5 & 0.42 \\
\hline \multirow{6}{*}{$3^{\#}$} & 0 & 0 \\
\hline & 1 & 0.35 \\
\hline & 2 & 1.25 \\
\hline & 3 & 1.80 \\
\hline & 4 & 3.45 \\
\hline & 5 & 0.80 \\
\hline
\end{tabular}

TABLE 4: The probability distributions of the durations (unit of time: day).

\begin{tabular}{lccc}
\hline$T_{1}$ & 6.5 & - & - \\
$p_{1}$ & 1 & - & - \\
$T_{2}$ & 9.5 & 10 & 10.5 \\
$p_{2}$ & 0.2 & 0.6 & 0.2 \\
$T_{3}$ & 8.8 & 9.6 & - \\
$p_{3}$ & 0.5 & 0.5 & - \\
$T_{4}$ & 9.3 & 10.2 & 10.8 \\
$p_{4}$ & 0.3 & 0.4 & 0.3 \\
\hline
\end{tabular}

TABle 5: The optimal selective maintenance decisions (unit of maintenance cost: $¥ 1,000$; unit of time: hour).

\begin{tabular}{|c|c|c|c|c|c|c|}
\hline \multirow[t]{2}{*}{$i$} & \multicolumn{3}{|c|}{$\begin{array}{c}\text { Scenario } 1 \\
\left.\text { Maintenance cost (level } l_{i, k}\right)\end{array}$} & \multicolumn{3}{|c|}{$\begin{array}{c}\text { Scenario } 2 \\
\left.\text { Maintenance cost (level } l_{i, k}\right)\end{array}$} \\
\hline & $k=1$ & $k=2$ & $k=3$ & $k=1$ & $k=2$ & $k=3$ \\
\hline 1 & $14.08(2)$ & $1.88(1)$ & $37.28(5)$ & $14.08(2)$ & $14.08(2)$ & $29.88(4)$ \\
\hline 2 & $40.31(5)$ & $40.31(5)$ & $17.61(2)$ & $34.91(4)$ & $32.31(3)$ & $17.61(2)$ \\
\hline 3 & $35.42(5)$ & $26.62(4)$ & $24.42(3)$ & $35.42(5)$ & $13.52(2)$ & $24.42(3)$ \\
\hline$R_{s, k+1}^{D}$ & 0.9846 & 0.8923 & 0.9672 & 0.9832 & 0.9357 & 0.9656 \\
\hline$C_{s, k}^{3, k+1}$ & 89.81 & 68.81 & 79.31 & 84.41 & 59.91 & 71.91 \\
\hline$\sum_{i=1}^{n, \kappa} t_{i, k}^{M}$ & 4.22 & 4.37 & 5.6 & 9.31 & 9.25 & 10.1 \\
\hline$C^{\diamond}$ & - & 237.93 & - & - & 216.23 & - \\
\hline$R_{s}$ & - & 0.8497 & - & - & 0.8883 & - \\
\hline
\end{tabular}

${ }^{*} C_{s, k}$ represents the total maintenance budget allocated to the $k^{\text {th }}$ maintenance activity; $C^{\diamond}$ represents the actual maintenance cost for all the missions.

\section{Summary and Conclusions}

In this paper, the conception of virtual age is used for the repairable components and the durations of the missions are regarded as discrete random variables, and a novel selective maintenance model under uncertainties and limited maintenance time is proposed. The imperfect maintenance based on the Kijima II model is introduced firstly, and then, the CDFs and PDFs of the virtual ages of components after a maintenance activity are derived. Subsequently, the survival probability of each component in different mission and the reliability of the system in successfully completing the missions are derived. Finally, the optimal selective maintenance decisions based on the genetic algorithm under limited maintenance budget and limited maintenance time are obtained. The proposed selective maintenance model takes the maintenance time into consideration, which is more consistent with the actual situation. In case of a limited maintenance time, the maintenance cost is larger and the reliability is worse, but they are sacrificed for saving time. The decisionmaker must make a compromise between the three.

In this paper, the states of each component are assumed to be binary variables and the durations of the missions are crisp values. It should be noted that the computation complexity will increase rapidly as the missions increase. Selective maintenance issues with respect to multistate 
systems as well as fuzzy durations will be studied in our future works.

\section{Data Availability}

The data used to support the findings of this study are included within the article.

\section{Disclosure}

The funders had no role in the design of the study; in the collection, analyses, or interpretation of data; in the writing of the manuscript; or in the decision to publish the results.

\section{Conflicts of Interest}

The authors declare that they have no conflicts of interest.

\section{Authors' Contributions}

H. G. and X. Z. conceptualized the study; H. G. developed the methodology; X. Z. provided software; H. G., X. Z., X. Y., and B. Z. validated the study; H. G. performed formal analysis; H. G. investigated the study; H. G. provided the resources; X. Z. was responsible for data curation; H. G. wrote and prepared the original draft; $\mathrm{H}$. G. wrote, reviewed, and edited the article; X. Z. visualized the study; X. Y. and B. Z. supervised the study; H. G. was involved in project administration; H. G. was responsible for funding acquisition. All authors have read and agreed to the published version of the manuscript.

\section{Acknowledgments}

This research was supported by the Sichuan Science and Technology Program, grant nos. 2019YJ0395 and 2021YJ0519 and General Aviation Special Research Project of Civil Aviation Flight University of China, grant no. THZX2018-09.

\section{References}

[1] B. D. Jonge and P. A. Scarf, "A review on maintenance optimization," European Journal of Operational Research, vol. 285, no. 3, pp. 805-824, 2019.

[2] X. Zhou, C. Wu, Y. Li, and L. Xi, "A preventive maintenance model for leased equipment subject to internal degradation and external shock damage," Reliability Engineering \& System Safety, vol. 154, pp. 1-7, 2016.

[3] Q. Qiu, L. Cui, and D. Kong, “Availability and maintenance modeling for a two-component system with dependent failures over a finite time horizon," Proceedings of the Institution of Mechanical Engineers, Part O: Journal of Risk and Reliability, vol. 233, no. 3, pp. 200-210, 2019.

[4] S. Alaswad and Y. Xiang, "A review on condition-based maintenance optimization models for stochastically deteriorating system," Reliability Engineering \& System Safety, vol. 157, pp. 54-63, 2017.

[5] S. Wu and M. J. Zuo, "Linear and nonlinear preventive maintenance models," IEEE Transactions on Reliability, vol. 59, no. 1, pp. 242-249, 2010.
[6] T. Nakagawa, Maintenance Theory of Reliability, Springer, Berlin, Germany, 2006.

[7] S.-H. Sheu, C.-C. Chang, Y.-L. Chen, and Z. George Zhang, "Optimal preventive maintenance and repair policies for multi-state systems," Reliability Engineering \& System Safety, vol. 140, pp. 78-87, 2015.

[8] J. H. Cha, M. Finkelstein, and G. Levitin, "Optimal mission abort policy for partially repairable heterogeneous systems," European Journal of Operational Research, vol. 271, no. 3, pp. 818-825, 2018.

[9] W. Si and Q. Yang, "A generalized mixed effect kijima model and application in optimal maintenance planning," IEEE Transactions on Reliability, vol. 65, no. 3, pp. 1551-1561, 2016.

[10] A. Yevkin and V. Krivtsov, "A generalized model for recurrent failures prediction," Reliability Engineering \& System Safety, vol. 204, p. 107125, 2020.

[11] L. Doyen, O. Gaudoin, and A. Syamsundar, "On geometric reduction of age or intensity models for imperfect maintenance," Reliability Engineering \& System Safety, vol. 168, pp. 40-52, 2017.

[12] Q. Qiu and L. Cui, "Optimal mission abort policy for systems subject to random shocks based on virtual age process," Reliability Engineering \& System Safety, vol. 189, pp. 11-20, 2019.

[13] A. Knezevic, L. Vasov, S. Vlacic, and C. Kostic, "Imperfect maintenance model for estimating aircraft fleet availability," Aircraft Engineering and Aerospace Technology, vol. 89, no. 2, pp. 338-346, 2017.

[14] Y. Liu, H. Z. Huang, and X. Zhang, "A data-driven approach to selecting imperfect maintenance models," IEEE Transactions on Reliability, vol. 61, pp. 101-112, 2011.

[15] W. F. Rice, C. R. Cassady, and J. A. Nachlas, "Optimal maintenance plans under limited maintenance time," in Proceedings of the 7th Industrial Engineering Research Conference, Banff, Canada, May 1998.

[16] C. R. Cassady, E. A. Pohl, and W. Paul Murdock, "Selective maintenance modeling for industrial systems," Journal of Quality in Maintenance Engineering, vol. 7, no. 2, pp. 104-117, 2001.

[17] C. R. Cassady, W. P. Murdock Jr., and E. A. Pohl, "Selective maintenance for support equipment involving multiple maintenance actions," European Journal of Operational Research, vol. 129, pp. 252-258, 2001.

[18] R. Rajagopalan and C. R. Cassady, "An improved selective maintenance solution approach," Journal of Quality in Maintenance Engineering, vol. 12, no. 2, pp. 172-185, 2006.

[19] C. M. La Fata and G. Passannanti, "A simulated annealingbased approach for the joint optimization of production/ inventory and preventive maintenance policies," The International Journal of Advanced Manufacturing Technology, vol. 91, no. 9-12, pp. 3899-3909, 2017.

[20] F. Moinian, H. Sabouhi, J. Hushmand, A. Hallaj, H. Khaledi, and M. Mohammadpour, "Gas turbine preventive maintenance optimization using genetic algorithm," International Journal of System Assurance Engineering and Management, vol. 8, no. 3, pp. 594-601, 2017.

[21] X. Chen, Y. An, Z. Zhang, and Y. Li, "An approximate nondominated sorting genetic algorithm to integrate optimization of production scheduling and accurate maintenance based on reliability intervals," Journal of Manufacturing Systems, vol. 54, pp. 227-241, 2020.

[22] M. Khatami and S. H. Zegordi, "Coordinative production and maintenance scheduling problem with flexible maintenance 
time intervals," Journal of Intelligent Manufacturing, vol. 28, no. 4, pp. 857-867, 2017.

[23] Z. Zhao, B. Xiao, N. Wang, X. Yan, and L. Ma, "Selective maintenance optimization for a multi-state system with degradation interaction," IEEE Access, vol. 7, pp. 99191-99206, 2019.

[24] Y. Liu and H. Z. Huang, "Optimal selective maintenance strategy for multi-state systems under imperfect maintenance," IEEE Transactions on Reliability, vol. 59, pp. 356-367, 2010.

[25] M. Pandey, M. J. Zuo, and R. Moghaddass, "Selective maintenance modeling for a multistate system with multistate components under imperfect maintenance," IIE Transactions, vol. 45, no. 11, pp. 1221-1234, 2013.

[26] Y. Liu, Y. Chen, and T. Jiang, "On sequence planning for selective maintenance of multi-state systems under stochastic maintenance durations," European Journal of Operational Research, vol. 268, no. 1, pp. 113-127, 2018.

[27] C. D. Dao and M. J. Zuo, "Optimal selective maintenance for multi-state systems in variable loading conditions," Reliability Engineering \& System Safety, vol. 166, pp. 171-180, 2017.

[28] T. Jiang and Y. Liu, "Selective maintenance strategy for systems executing multiple consecutive missions with uncertainty," Reliability Engineering \& System Safety, vol. 193, p. 106632, 2020.

[29] M. Pandey, M. J. Zuo, R. Moghaddass, and M. K. Tiwari, "Selective maintenance for binary systems under imperfect repair," Reliability Engineering \& System Safety, vol. 113, pp. 42-51, 2013.

[30] T. Jiang and Y. Liu, "Robust selective maintenance strategy under imperfect observations: a multi-objective perspective," IISE Transactions, vol. 52, no. 7, pp. 751-768, 2020.

[31] A. Khatab, E. H. Aghezzaf, I. Djelloul, and Z. Sari, "Selective maintenance optimization for systems operating missions and scheduled breaks with stochastic durations," Journal of Manufacturing Systems, vol. 43, pp. 168-177, 2017.

[32] C. Diallo, U. Venkatadri, A. Khatab, and Z. Liu, "Optimal selective maintenance decisions for large serial $k$-out-of- $n$ : $G$ systems under imperfect maintenance," Reliability Engineering \& System Safety, vol. 175, pp. 234-245, 2018.

[33] D. T. Nguyen, Y. Dijoux, and M. Fouladirad, "Analytical properties of an imperfect repair model and application in preventive maintenance scheduling," European Journal of Operational Research, vol. 256, no. 2, pp. 439-453, 2017.

[34] A. Khatab and E.-H. Aghezzaf, "Selective maintenance optimization when quality of imperfect maintenance actions are stochastic," Reliability Engineering \& System Safety, vol. 150, pp. 182-189, 2016.

[35] H. Li, H.-Z. Huang, Y.-F. Li, J. Zhou, and J. Mi, "Physics of failure-based reliability prediction of turbine blades using multi-source information fusion," Applied Soft Computing, vol. 72, pp. 624-635, 2018.

[36] M. Yazdi, S. Daneshvar, and H. Setareh, "An extension to fuzzy developed failure mode and effects analysis (FDFMEA) application for aircraft landing system," Safety Science, vol. 98, pp. 113-123, 2017.

[37] M. Yazdi, S. Kabir, and M. Walker, "Uncertainty handling in fault tree based risk assessment: state of the art and future perspectives," Process Safety and Environmental Protection, vol. 131, pp. 89-104, 2019.

[38] H. Li, H. Diaz, and C. G. Soares, "A developed failure mode and effect analysis for floating offshore wind turbine support structures," Renewable Energy, vol. 164, pp. 133-145, 2020.
[39] M. Yazdi, F. Nikfar, and M. Nasrabadi, "Failure probability analysis by employing fuzzy fault tree analysis," International Journal of System Assurance Engineering and Management, vol. 8, no. 2, pp. 1177-1193, 2017.

[40] M. Yazdi, A. Nedjati, E. Zarei, and R. Abbassi, "A novel extension of DEMATEL approach for probabilistic safety analysis in process systems," Safety Science, vol. 121, pp. 119-136, 2020.

[41] H. Li, A. P. Teixeira, and C. Guedes Soares, "A two-stage failure mode and effect analysis of offshore wind turbines," Renewable Energy, vol. 162, pp. 1438-1461, 2020.

[42] H. Li, C. Guedes Soares, and H.-Z. Huang, "Reliability analysis of a floating offshore wind turbine using Bayesian networks," Ocean Engineering, vol. 217, p. 107827, 2020.

[43] M. Yazdi and S. Kabir, "A fuzzy Bayesian network approach for risk analysis in process industries," Process Safety and Environmental Protection, vol. 111, pp. 507-519, 2017. 